\title{
A Survey on the Duration of Leakage and Patient Perceived Bottlenecks to Seeking Fistula Treatment in Uganda
}

\author{
Asiimwe Ian Shane ${ }^{1}$ Matovu Alphonsus ${ }^{2}$ \\ ${ }^{1}$ Hoima Regional Referral Hospital \\ ${ }^{2}$ Mubende Regional Referral Hospital, Uganda
}

DOI: http://dx.doi.org/10.3126/njog.v13i2.21711

\begin{abstract}
Aims: Uganda still has high fistula burden with 75000-100000 women still living with obstetric fistula and yet new cases are being formed. The ministry of health together with supporting partners established regional treatment centers among which includes Hoima and Mubende regional referral hospitals. This aimed to assess the duration of leaking urine and patient perceived reasons for delay to seek repair among genitourinary fistula mothers who presented in fistula camps at Mubende and Hoima fistula treatment centers.
\end{abstract}

Methods: This was a retrospective study in which qualitative and quantitative data was collected from standardized patient records of patient chats and registers filled by fistula surgeons.

Results : We had a total of 125 mothers from 19 districts and of age between 16 to 72 years with genitourinary fistula operated. The majority (47.4\%) of the clients were 25 years or less, of low parity with $41 \%$ (para $1+0)$ and without formal employment. Only $59.5 \%$ of the clients presented within one year from the time they developed the fistula. Among the patient perceived bottlenecks are the perception that fistulas are only treated during camps, lack of money, ill health resulting from difficult labour and lack of awareness that the bladder can be repaired.

Conclusions: With the low turn up of mothers seeking repair, there is need to reduce leaking time and lessen suffering of obstetric fistula patients by creating more awareness, promoting routine repairs and continued financial support to care for fistula clients.

Keywords: leaking urine, obstetric fistula, repair of fistula.

\section{INTRODUCTION}

Uganda is among the countries with high fistula burden with 750000-100000 mothers still living with obstetric fistula. ${ }^{1}$ This study looks at the duration of leakage and patient perceived bottlenecks to seeking fistula treatment in Hoima and Mubende fistula treatment centers from June 2017 to July 2018. The awareness of fistula signs among Ugandan women ages $15-49$ is $61 \%$ and $74 \%$ for rural and urban areas respectively ${ }^{1}$, however fistula clients seem to wait for camps to seek repair. The average duration of leakage is still long 6 to 5 years $^{2-4}$ with a variable proportion of clients $(42 \%$ to $80 \%)$ receiving repair within one year of developing the fistula. ${ }^{3,5}$ The optimal timing for repair is still debatable and early repair could compromise success of the surgery. ${ }^{6}$ This study was guided by the three delays model $^{7}$ with focus on the patients' perceived reasons as these opinions directly influence the compliance to seek treatment.

\section{CORRESPONDENCE}

Dr Asiimwe Ian Shane

Hoima Regional Referral Hospital, Uganda

Email: ianshaneasiimwe@yahoo.com

\section{METHODS}

This was a retrospective study in which qualitative and quantitative patient data was collected from hospital patient records. Permission to access patient hospital records was obtained from both institutions. We examined hospital records (standard patient forms, hospital entry registers) of all fistula patients clerked by a fistula surgeons during the fistula camps from June 2017 to July 2018. Fistulas were classified using the Kees Waljick 1995 classification. The study was conducted in Hoima (mid-western Uganda) and Mubende (central Uganda) regional referral hospitals. Both hospitals have a resident fistula surgeon who is capable of repairing obstetric fistulas. Hoima regional referral hospital is 280 bed capacity hospital and serves 7 districts and conducts 3-4 fistula camps annually with support from donor partners while Mubende regional referral hospital a 175 bed capacity hospital is a referral hospital for 6 districts and carries out 3-4 fistula camps every year with support from donor partners.

Our study population consisted of all genitourinary fistula patients operated in fistula camps in Hoima and Mubende regional referral hospitals between June 2017 and July 2018. Our variables were patient 
demographics (age, home district, parity, marital status, occupation), type of genitourinary fistula), duration of leakage in months and patients' perceived reasons for delay to seek repair for the fistula. Data was collected, entered into excel and cleaned to avoid duplication of cases for those who came for multiple repairs and exported to SPSS 18 for analysis. Qualitative data was categorized into themes and presented in form of graphs and narrative form and quantitative data summarized into graphs and narrative form.

\section{RESULTS}

A total of 125 mothers with genitourinary fistula were operated, of age 16 years to 72 years. The majority of the patients $(47.4 \%)$ were 25 years or less, of low parity with $41 \%$ (para $1+0$ ) and 20\% (para 2+0). Majority of the clients are low income earners, mainly peasant farmers $(77.6 \%)$. They came from 19 districts and most of them come from Mubende (29.6\%), Hoima (20\%) and Kibale (15\%).

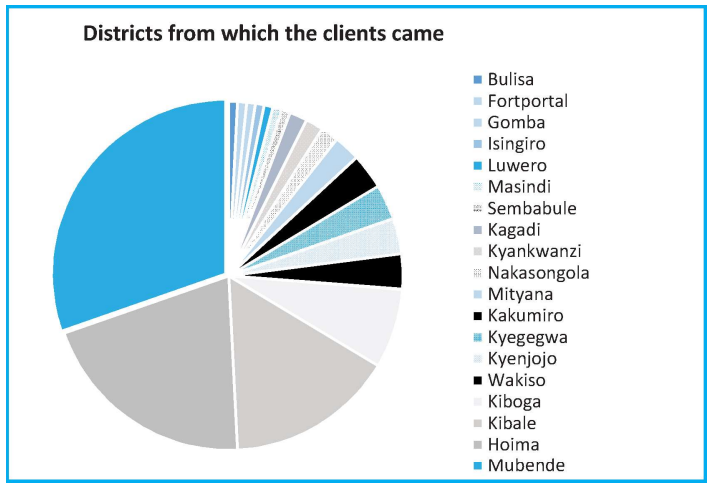

Figure 1: Distribution of clients according to districts.

The average duration of leakage of 41 month with $59.5 \%$ of the clients presenting within one year from the time they developed the fistula. There were 71.6 $\%$ of the clients seeking fistula treatment within the two years. The earliest presentation was at 2 weeks and the longest duration of leakage was 35 years.

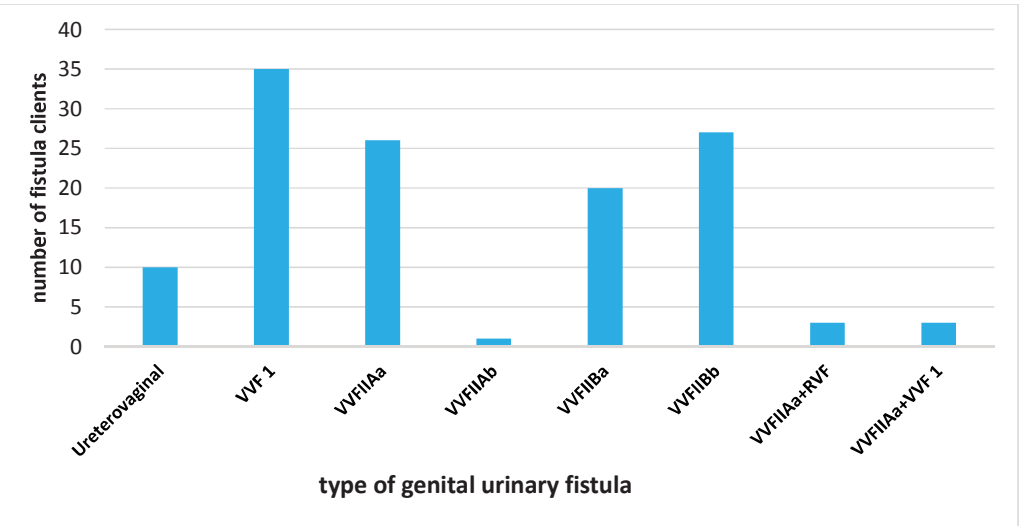

Figure 2: Urogenital fistula repaired at Hoima and Mubende Hospitals.

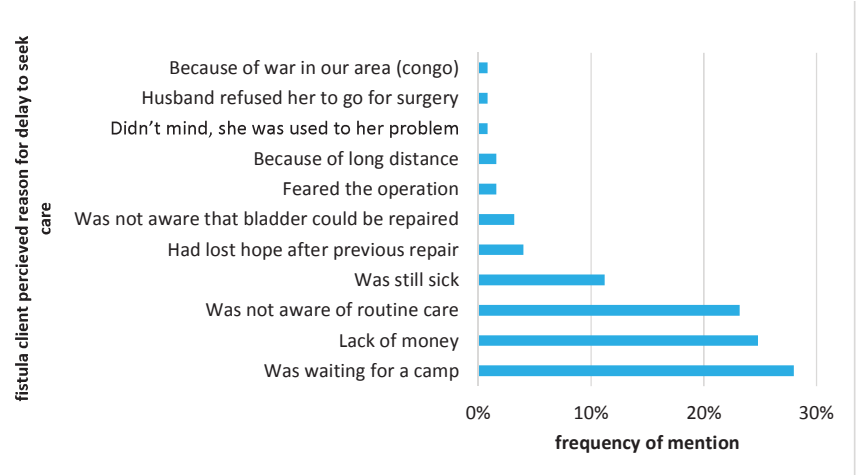

Figure 3: Fistula Clients' Perceived Reasons for Delay to Seek Repair. 


\section{DISCUSSION}

There is a large proportion of obstetric fistula patients with long duration of leakage and most of these are the young productive age group. Whereas we see more numbers seeking for care within the first one year of developing urogenital fistula, this could be attributed to the routine quarterly fistula camps at the treatment centers. This turn up however is still low considering the amount of suffering the obstetric fistula causes.

The study highlights the significant contribution of routine camps to reducing duration of leakage and emphasizes that obstetric fistula is not in isolation but coupled with other ailments adding to suffering. The obstetric fistula is only part of the obstructed labour injury complex ${ }^{8}$ and patients may still be unfit for surgery.
The problem of financial limitations has been reported in other studies ${ }^{1,9}$, similar to our findings. With little income obstetric fistula clients will continue to delay to seek repair.

\section{CONCLUSIONS}

We still have a bulk of mothers leaking urine for more than a year. There is need to reduce the duration of leakage and alleviate suffering of obstetric fistula mothers through continued efforts to create more awareness and hope for fistula mothers, encouraging obstetric fistula clients to seek repair early and promoting routine repairs. This calls for continued financial support to care for obstetric fistula clients to enable them access specialist care.

\section{REFERENCES}

1. Uganda Bureau of Statistics -UBOS and ICF. Uganda Demographic and Health Survey 2016. Kampala, Uganda: UBOS and UCIF; 2018.p 99-163. https://www.dhsprogram. com/pubs/pdf/FR333/FR333.pdf

2. Hancock B, Collie M. Vesico-vaginal fistula surgery in Uganda. East and Central African Journal of surgery. 2004;9(2):32-7. https://www.ajol.info/index.php/ecajs/article/ view/137248

3. McCurdie FK, Moffatt J, Jones K. Vesicovaginal fistula in Uganda. Journal of Obstetrics and Gynaecology. 2018; 38(6): 822-827. https://doi.org/10.1080/01443615.2017.1407301

4. Morren G, van den Boogaard W, Dominguez E. Management of Obstertic Fistula in Burundi. Bruxelles, Belgium: MSFODB medical department; May 2016. p9-29. http://hdl. handle.net/10144/610799

5. Weston K, Mutiso S, Mwangi JW, Qureshi Z, Beard J, Venkat P. Depression among women with obstetric fistula in Kenya. International Journal of Gynecology \& Obstetrics. 2011;115(1):31-3.https://doi.org/10.1016/j.ijgo.2011.04.015
6. Kumar S, Kekre NS, Gopalakrishnan G. Vesicovaginal fistula: An update. Indian journal of urology. 2007;23(2):187-91. http:/www.indianjurol.com/text.asp?2007/23/2/187/32073

7. Thaddeus S, Maine D. Too far to walk: maternal mortality in context. Social Science and Medicine. 1994;38(8):1091-110. https://pdfs.semanticscholar.org/b417/80532a0b5d28547b1f6 59e64dacf36eac98d.pdf

8. Wall L L, Arrowsmith S D, Briggs N D, Browning A, Lassey A. The obstetric vesicovaginal fistula in the developing world. Obstetrical and Gynecological Survey. 2005;60(7):S3-51. https://www.ics.org/Publications/ICI_3/v2.pdf/chap22.pdf

9. Keya KT, Sripad P, Nwala E, Warren CE. Poverty is the big thing: exploring financial, transportation, and opportunity costs associated with fistula management and repair in Nigeria and Uganda. International Journal for Equity in Health. 2018;17:70. https://doi.org/10.1186/s12939-018-0777-1 